Voix et Images

volxetimages

\title{
Mélancolie, nostalgie et disparition
}

Pascal Riendeau

Volume 39, numéro 1 (115), automne 2013

URI : https://id.erudit.org/iderudit/1022999ar

DOI : https://doi.org/10.7202/1022999ar

Aller au sommaire du numéro

Éditeur(s)

Université du Québec à Montréal

ISSN

0318-9201 (imprimé)

1705-933X (numérique)

Découvrir la revue

Citer ce compte rendu

Riendeau, P. (2013). Compte rendu de [Mélancolie, nostalgie et disparition].

Voix et Images, 39(1), 145-150. https://doi.org/10.7202/1022999ar

Ce document est protégé par la loi sur le droit d'auteur. L'utilisation des services d'Érudit (y compris la reproduction) est assujettie à sa politique d'utilisation que vous pouvez consulter en ligne.

https://apropos.erudit.org/fr/usagers/politique-dutilisation/ 


\title{
R O M A N
}

\author{
Mélancolie, nostalgie et disparition \\ $+++$
}

PASCAL RIENDEAU

Université de Toronto

Pierre Samson aime bien bousculer le milieu littéraire québécois : en 2000, son court essai Alibi ${ }^{1}$ ne ménageait pas la critique de ce petit monde, alors que sept ans plus tard son roman Catastrophes en offre, entre autres choses, une satire. Le travail se poursuit dans Lettres crues, échange épistolaire avec le poète Bertrand Laverdure dans lequel Samson développe sa pensée sur le sujet avec ironie ou sarcasme, mais rarement de manière virulente. La parole du Samson essayiste est souvent emportée, elle peut paraître injuste, elle agace et irrite sans doute, mais elle demeure salutaire. Samson se défend pourtant d'être un véritable essayiste ; il répète qu'il est romancier et qu'un romancier ne devrait pas consacrer un essai à son écriture, à sa propre œuvre. Dans Alibi, il ajoute même: «je répugne à expliquer ma vision artistique» (21).

L'art romanesque de Pierre Samson s'exprime dans tous les aspects de son œuvre. Rien n'est négligé, à commencer par le titre. Celui de son dernier roman nous entraîne dans un univers singulier, car La maison des pluies ${ }^{2}$ ne représente pas qu'une métaphore inhabituelle pour le simple nuage; c'est surtout un voyage vers le passé, là où la mélancolie et la nostalgie guettent. Benjamin Paradis revient au pays après avoir longtemps vécu à l'étranger, explorant les continents lors d'enquêtes sur des groupes de locuteurs dont la langue risque de disparaître. Linguiste, Benjamin a trouvé un poste dans une université montréalaise où il donne notamment un cours sur les «langues en péril ». Son retour définitif à Montréal, sa ville natale, lui permet de revoir des personnages de son passé qui peuvent l'aider à élucider sa situation actuelle plutôt inusitée. Un ancien camarade de classe qu'il retrouve grâce à un ami apprend à Benjamin qu'un jeune homme, qui est peut-être son fils, est à sa recherche. À son tour, Benjamin tente de savoir qui est cet étranger qui suit sa trace, accumule des informations sur lui, mais ne semble pas vouloir le rencontrer. Cette quête, qui occupera de plus en plus les pensées de Benjamin, devient l'occasion pour lui de redécouvrir des pans oubliés de son passé. Le narrateur multiplie les différents récits et cultive l'art de la digression afin de briser la linéarité du roman. Le romancier ironique et

$$
+++
$$

1 Pierre Samson, Alibi, Montréal, Leméac, coll. «Ici l'ailleurs», 2000, 103 p. 2 Pierre Samson, La maison des pluies, Montréal, Les Herbes rouges, 2013, 267 p. 
l'essayiste romanesque se rejoignent ici : «Un roman vit et respire grâce à ses détours qui donnent au récit son oxygène, nourrissent ses muscles ${ }^{3} "$, affirme Samson. C'est d'ailleurs de cette manière qu'il mène la narration de La maison des pluies. Ce refus de la ligne droite incite donc le narrateur à emprunter toutes sortes de pistes, et les arrêts s'allongent parfois. Ainsi, il redécouvre une nouvelle écrite à l'adolescence qu'il avait complètement oubliée. "Automne» a été publiée, se rappelle-t-il alors, dans une «bonne revue». Il a pu la relire en se rendant à la Grande Bibliothèque - dont le projet architectural est l'objet de sarcasmes dans le roman - après avoir suivi le parcours de celui que l'on prend pour son fils. Entièrement reproduite, la nouvelle se distingue nettement du style de Samson, c'est-à-dire que l'auteur a réussi à composer une véritable "fausse nouvelle» de jeunesse, en maîtrisant parfaitement un style simple et hésitant. En revanche, on constate dans cette nouvelle, outre la nostalgie de l'adolescent qui raconte sa première rentrée scolaire (à six ans), un véritable intérêt pour la précision du vocabulaire et même un goût un peu précieux pour les mots rares ou recherchés.

Pierre Samson n'est pas qu'un habile créateur de narrations complexes où les récits s'enchevêtrent; c'est également un vrai styliste. Peu de romanciers québécois contemporains montrent une telle maîtrise de la langue, du vocabulaire et des divers registres linguistiques. Son style intègre bien l'humour et l'ironie, mais il suggère aussi un véritable plaisir d'approfondir les combinaisons lexicales: «au dictionnaire, mes chéris, la langue au grand complet nous appartient, alors explorons-la ${ }^{4}$ ", écrit Samson à son correspondant Laverdure. Dans l'ensemble, la narration de La maison des pluies reste sobre, mais certains passages montrent des élans stylistiques bien maîtrisés, comme lorsqu'il s'agit de décrire son ancien camarade, un homme "que des décennies de laisser-aller ont empâté jusqu'à créer un gros patapouf désormais avachi dans un fauteuil trop beau pour lui, une outre passée au stade de citerne molle et malodorante, emmaillotée dans des couches et des couches de tee-shirts, de chemises et de débardeurs chiffonnés [...]» (56). Il faut préciser que c'est surtout dans les (trop) longs dialogues qu'apparaissent des mouvements plus baroques, en particulier quand la parole incombe à Shirley, dit «Big Daddy», un ami de Benjamin qui a «accumulé et nourri un savoir quasiment encyclopédique de choses parfaitement futiles» (35). Ce n'est d'ailleurs sans doute pas un hasard si ce personnage haut en couleur, le plus loquace du roman, celui qui se présente comme un véritable amoureux de «la langue de Molière", langue qu'il manie avec une telle verve, une telle originalité, est un anglophone:

Putain, j'envoie des lettres de protestation à Radio-Canada qui passe la grammaire à la tronçonneuse. Le français, moi, I love it! Mais eux, c'est-à-dire les candidats aux coïts télécommandés, ils ne contrôlent ni leur syntaxe ni leur amour-propre. [...] Ils ne deviennent qu'une queue à tripoter, qu'un sphincter à distendre, qu'une peau de zèbre ou de rat musqué à pétrir avec une amabilité parfaitement optionnelle. (48)

$$
+++
$$

3 Pierre Samson et Bertrand Laverdure, Lettres crues, Montréal, La Mèche, coll. «L'ouvroir», 2012, p. 236.

4 Ibid., p. 293. 
Se lancer dans une critique de la langue française parlée ou écrite au Québec ne représente rien de très nouveau ni de très original. Samson lui-même y participe volontiers dans ses essais. Heureusement, cette envolée de Big Daddy mise davantage sur l'humour et évacue ainsi la lourdeur possible de tels discours dans un roman.

De nombreux autres personnages que Benjamin revoit ou dont il narre un épisode de vie viennent complexifier le récit: sa mère, son cousin, son oncle, Odile Le Doaré - l'enseignante qui l'a encouragé à écrire - , sans oublier deux femmes dont une peut être la mère du jeune homme mystérieux, à savoir Kim, la Néerlandaise rencontrée au Japon vingt ans plus tôt, ou Béatrice, avec qui il a connu un épisode amoureux torride à Montréal un peu avant. Mais le roman de Pierre Samson ne possède pas de véritable dénouement: jusqu'à la fin, La maison des pluies glisse et nous entraîne vers un autre récit, rejetant ainsi toute résolution de l'intrigue.

Si on apprécie les romans de Samson pour la virtuosité stylistique qui s'y déploie, on ne peut pas en dire autant des romans de Claude Jasmin. Leur pertinence se trouve ailleurs. Dans Anita, une fille numérotée ${ }^{5}$, Jasmin privilégie une écriture simple, directe, qui mélange habilement l'humour et la gravité. L'intérêt principal réside surtout dans l'absence de complaisance de ce petit roman autobiographique. L'auteur raconte son histoire d'amour pour une jeune femme juive dans le Montréal très catholique de l'époque. Le récit se déroule en 1950-1951, mais Jasmin ne semble pas très préoccupé par l'exactitude des dates ou des événements spécifiques. Il reconstruit l'univers de cette période, sans que la temporalité soit toujours très précise. On peut même situer l'histoire un an plus tôt si l'on se fie à la quatrième de couverture, où il est question du récit de l'auteur, né en 1930, qui a commencé ses études à l'École du meuble à 18 ans. À la suite de résultats scolaires désastreux, le narrateur voit les portes de l'université se fermer devant lui et accepte la proposition de son père de s'inscrire à une école de métiers afin d'y apprendre les arts céramiques. C'est là qu'il rencontre Anita Geller, jeune Juive d'origine polonaise rescapée d'Auschwitz, dont presque toute la famille a été décimée. Elle habite maintenant à Montréal avec son père, après avoir brièvement vécu en France.

Une histoire d'amitié, puis d'amour se développe entre le narrateur et la belle Anita, dans cette ville qui s'ouvre tranquillement au monde et donne l'impression d'être un lieu agréable pour les jeunes qui découvrent la vie artistique et une certaine «bohème». Le narrateur continue de fréquenter Anita, malgré les préjugés tenaces dont fait preuve sa famille à lui, surtout son père, qui lui répète que les Juifs ne font pas partie du même monde qu'eux. C'est par hasard qu'Anita et le père du narrateur vont un jour se rencontrer. Constatant une animosité évidente de sa part, Anita ose lui demander:

- En fait, qu'est-ce que vous nous reprochez au juste, à nous, les Juifs, pourquoi nous détestez-vous?

5 Claude Jasmin, Anita, une fille numérotée, Montréal, XYZ éditeur, 2013, 187 p. 


\section{$[\ldots]$}

- Vous voulez savoir ce que les catholiques du monde entier vous reprochent? Vous allez le savoir. Vous avez tué le Christ, notre Seigneur Jésus-Christ! Ne l'oubliez jamais.

Un long silence. Anita est secouée, muette de stupéfaction [...]. Je ne sais plus où me mettre. Forte envie d'aller gifler mon père. Satisfait, papa marche vers la sortie.

Dans son récit, Jasmin a su retransmettre cette ambiance de l'antisémitisme ordinaire qui régnait dans le Québec des années Duplessis, en montrant son père en bon catholique satisfait et mesquin, convaincu qu'il a raison, que ses préjugés sont fondés. Jasmin a eu l'habileté de bien décrire les émotions du jeune homme à l'égard de son père (honte, mépris) et de son amie (tristesse, compassion), mais n'a pas ajouté de jugement supplémentaire. Autrement dit, aucune voix morale d'aujourd'hui ne vient s'imposer afin de porter un regard a posteriori sur l'épisode.

Le numéro tatoué sur le bras d'Anita s'explique par son internement au camp d'Auschwitz, le narrateur le comprend, même s'il avoue ne pas savoir grand-chose de tout ce qui s'est passé dans les camps de concentration. S'il n'ose pas lui poser de questions sur ce sujet, il croit néanmoins que le tatouage d'Anita la classe dans une catégorie à part, celle des prostituées. Le narrateur apprendra trop tard que cette histoire n'est qu'une invention des Gladstone, voisins juifs des Geller n'aimant pas le père d'Anita, lui qui avait eu l'audace de rejeter Dieu et la religion. Sans jamais expliquer le phénomène, le narrateur ne propose pas non plus de comparaison simple entre une haine des Juifs (celle de son père) qui s'inscrit dans une idéologie bien précise et une haine à l'égard de celui qui a renoncé au judaïsme (celle des voisins). Les conséquences de ces attitudes haineuses font en sorte que le narrateur s'éloigne d'Anita, jusqu'à ce qu'elle quitte définitivement Montréal pour Toronto.

À l'évidence, le souci d'une reconstitution historique très précise importe peu, ce qui explique la présence de quelques anachronismes. Par exemple, comment le narrateur, lors de ses élans créatifs, aurait-il pu avoir été influencé par le théâtre de Beckett dès 1950, alors que les premières représentations d'En attendant Godot datent de janvier 1953 ? L'anachronisme le plus surprenant survient toutefois durant la tirade du père d'Anita contre Dieu et la religion, qui se termine par une intrigante suggestion de lecture : Si c'est un homme de Primo Levi, dont la traduction française n'a pourtant été publiée qu'en 1987. Dans l'économie du récit, cela ne paraît pas très grave, car ces exemples semblent plutôt choisis pour leur force d'évocation, afin d'éclairer la situation ou la pensée d'un personnage. À l'exception de l'envolée du père d'Anita, les dialogues du roman demeurent plutôt brefs et ne laissent pas de place à une exploration langagière. Jasmin privilégie un style sans fioritures. On pourrait lui reprocher cette pauvreté stylistique, mais il faut bien admettre que cette sobriété - les phrases contiennent de six à quinze mots, rarement plus de vingt — sied parfaitement à Anita, une fille numérotée. Jasmin semble avoir trouvé le ton juste pour raconter une histoire de jeunesse qui va bien au-delà du roman autobiographique en incluant sur un mode mineur une critique de la société québécoise très catholique de l'époque. L'échec de l'histoire d'amour du narrateur et d'Anita nous donne un récit réussi et pertinent plus 
de soixante ans plus tard. Malgré le dénouement terrible raconté dans l'épilogue, malgré son constat que «le mal existait. Vraiment» (185), la fin nous laisse entrevoir un espoir grâce à l'art. Sur cette toile de Jean-Paul Lemieux que le narrateur contemple dans les derniers instants se trouve une femme seule sur qui se superpose le visage d'Anita, le regardant d'un sourire beau et triste.

Villes mortes ${ }^{6}$ de Sarah Berthiaume est présenté par son éditeur comme un recueil de nouvelles. Il s'agit plutôt de quatre courts textes dramatiques « aménagés» en quelque sorte pour la publication. Ils ont été présentés dans leur version définitive à la salle Jean-Claude-Germain du Théâtre d'Aujourd'hui en avril 2011. Légèrement narrativisés, les textes étaient d'abord des monologues interprétés au théâtre par quatre comédiennes différentes, dont Sarah Berthiaume. Les quatre «villes mortes» - qui, au demeurant, ne sont pas toutes mortes ni toutes des villes - où se déroulent les nouvelles sont Pompéi, Gagnonville (on dit plus simplement «Gagnon»), Kandahar et le «quartier» DIX30 à Brossard. Les nouvelles sont accompagnées d'illustrations qui accentuent les aspects ironiques ou dérisoires des situations ${ }^{7}$. On le voit, si les deux premières villes ont effectivement disparu, la troisième est encore bien vivante, quoiqu'elle soit aux prises avec un conflit armé qui s'éternise et une occupation militaire étrangère pour le moins ambivalente. Quant au dynamique DIX30, il représente la nonville, ce lieu inhabitable. L'humour et l'ironie dominent dans les textes de Berthiaume, et le style reste fortement marqué par une oralité spécifiquement théâtrale. On n'est jamais très loin de la reproduction de cette parole directe, immédiate de la scène, la narratrice se plaisant à interpeller son lecteur telle une actrice s'adressant à son public.

Chaque histoire est racontée à la première personne par une narratrice différente : l'amante qu'on laisse tomber, la fille aux parents nostalgiques qui ont dû abandonner leur ville nord-côtière, l'actrice au chômage qui accepte de jouer dans un film étudiant, la jeune mère célibataire qui s'exile à Kandahar pour travailler chez Tim Hortons. La narratrice de «Pompéi» est une jeune femme trompée par son conjoint lors de son séjour à Naples. C'est après l'avoir rejoint dans cette ville qu'elle apprend la nouvelle et exprime son désarroi :

Sa bouche continue de cracher des mots sur moi. Hasard, collègue, jamais pensé, Italienne, début du mois, coup de foudre, lâche, téléphone, courriel, pas capable, comprendre, excuses, pardonner, excuses, fini, excuses. Chaque mot est une braise rouge qui passe de son ventre à mon cœur [...]. (19)

Ailleurs, le style est plus joualisant et le franglais abonde. Il faut dire que l'efficacité de la parole théâtrale se traduit parfois mal en langage narratif. En revanche,

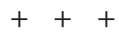

6 Sarah Berthiaume, Villes mortes, Montréal, Les éditions de ta mère, 2013, 101 p. 7 Chaque nouvelle est illustrée par un artiste différent: Sébastien Thibault («Pompéi »), François Léveillé (« Gagnonville»), Gabrielle Laila Tittley («Kandahar») et Benoit Tardif («DIX30»). 
Berthiaume ne se contente pas de raconter des histoires de femmes en difficulté; elle propose une critique sociale pertinente. Que ce soit sur les bonnes intentions de l'armée canadienne visant à libérer les femmes afghanes ou sur les joies de l'hyperconsommation que l'on trouve au DIX30, la parole est juste, l'humour grinçant. Il manque certainement un approfondissement pour transposer ces monologues en véritables œuvres narratives structurées et cohérentes, mais le ton, l'humour et la critique montrent une parole judicieuse.

Claude Jasmin, Pierre Samson et Sarah Berthiaume, trois auteurs autodidactes appartenant à trois générations différentes, ont exploré ici la mélancolie, la nostalgie ou la disparition. Pour le protagoniste du roman de Samson, la mélancolie est un sentiment qu'il faut éviter, que l'on doit même fuir. Le récit de Jasmin, au contraire, nous laisse croire à un certain plaisir mélancolique ou plutôt à un recours aux sentiments mélancoliques ou nostalgiques comme moteur de la construction du récit. Ni la mélancolie ni la nostalgie n'accompagnent les narratrices des récits de Berthiaume, même si elles deviennent parfois le témoin d'un monde révolu, une disparition qui mène alors à la création d'univers parallèles fantaisistes. 